\title{
Scalar field spacetimes and the anti-de Sitter space/conformal-field theory conjecture
}

\author{
Saurya Das* \\ Department of Physics, The University of Winnipeg, 515 Portage Avenue, Winnipeg, Manitoba, Canada R3B 2E9 \\ J. Gegenberg ${ }^{\dagger}$ and V. Husain ${ }^{\ddagger}$ \\ Department of Mathematics and Statistics, University of New Brunswick, Fredericton, New Brunswick, Canada E3B 5A3
}

(Received 6 February 2001; published 28 August 2001)

\begin{abstract}
We describe a class of asymptotically AdS scalar field spacetimes, and calculate the associated conserved charges for three, four and five spacetime dimensions using the conformal and counterterm prescriptions. The energy associated with the solutions in each case is proportional to $\sqrt{M^{2}-k^{2}}$, where $M$ is a constant and $k$ is a scalar charge. In five spacetime dimensions, the counterterm prescription gives an additional vacuum (Casimir) energy, which agrees with that found in the context of AdS conformal-field theory (CFT) correspondence. We find a surprising degeneracy: the energy of the "extremal" scalar field solution $M=k$ equals the energy of global AdS. This result is discussed in light of the AdS/CFT conjecture.
\end{abstract}

DOI: 10.1103/PhysRevD.64.065027

PACS number(s): 11.10.Kk, 11.25.Mj

The nonlinear coupling of gravity to matter in general relativity presents difficult technical problems in attempts to understand gravitational interactions of elementary particles and strings, as well as questions such as the details of gravitational collapse. Progress in the former area has come mainly from treating quantum fields as propagating on fixed background geometries [1], whereas much of the progress in the latter has come from detailed numerical work [2].

Exact solutions of the relevant matter-gravity equations can play an important role by shedding light on questions of interest in both general relativity and string theory. One is often interested in certain classes of solutions, with specified asymptotic properties, the most common of which are the asymptotically flat spacetimes. Recent work in string theory however, has highlighted the importance of another class of spacetimes via the AdS/conformal-field theory (CFT) conjecture [3]. These are the asymptotically anti-de Sitter spacetimes (AAdS).

The AdS/CFT conjecture is a duality between string theory on $\mathrm{AdS}_{5} \times \mathrm{S}^{5}$ and the large $N$ limit of conformally invariant $\mathcal{N}=4 S U(N)$ Yang-Mills (YM) theory on the boundary of $\mathrm{AdS}_{5}$. This conjecture proposes a direct correspondence between physical effects associated with fields propagating in AdS spacetime and those of a conformal quantum field theory on the boundary of AdS spacetime. Significant evidence for the conjecture has come from studying free scalar or other fields on a fixed AdS background [4]. Another important aspect of this conjecture is that it connects infrared effects in AdS space to ultraviolet effects in the boundary theory [5]. This in turn implies a connection between low and high energy physics in the respective theories. A fully non-linear gravity-scalar field solution can provide a window into this aspect of the conjecture as well, as is the case for scalar fields on a fixed background.

\footnotetext{
*Email address: saurya@theory.uwinnipeg.ca

†Email address: lenin@math.unb.ca

‡Email address: husain@math.unb.ca
}

With this motivation we study the Einstein-scalar field system, with minimally coupled massless scalar field and negative cosmological constant. We present static spherically symmetric AAdS solutions of these equations. For spacetime dimension $d=3$, the equations can be solved exactly. For $d$ $\geqslant 4$, the corresponding equations can be solved analytically for large radial distance, i.e., asymptotically. We calculate conserved charges associated to these spacetimes using the conformal [6] and the counterterm [7] prescriptions. Finally we discuss some consequences of our results, in particular the surprising energy degeneracies associated with the solutions: The "extremal" limit of our solutions have the same energy as the corresponding global $\mathrm{AdS}_{d}$ spacetime.

The solutions we discuss are singular at the origin. This raises the question of whether these are "admissable" in the context of the AdS/CFT conjecture. The fact that the energy of this class of solutions turns out to be finite is a hint that the singularity may be resolved by quantum effects. Indeed, there has been a suggestion [8] that singularities are valuable in that they are concomitant with the absence of an energy lower bound, which is another criteria for excluding solutions. This is not the case for the solutions we present, since the corresponding energies are well defined and bounded below by the energy of global AdS.

The equality of the vacuum energy of AdS (calculated via the counter-term method), and the Casimir energy of the boundary YM theory is considered to be a piece of the evidence for the AdS/CFT conjecture. However, our degeneracy result demonstrates that there is another spacetime with the same Yang-Mills Casimir energy. This raises an ambiguity concerning this dictionary entry of the AdS/CFT correspondence.

The $d=3$ matter-gravity model considered in this paper has been studied numerically in the full time dependent context with circular symmetry in Refs. $[9,10]$, where critical behavior at the onset of black hole formation is observed. More recently the critical exponent for the apparent horizon radius found in [10] has been verified by an analytical perturbation theory calculation [11]. 
We consider the massless scalar field minimally coupled to gravity in $d$ spacetime dimensions. With the parametrization of the negative cosmological constant as $\Lambda=$ $-(d-1)(d-2) / 2 l^{2}$, the field equations are

$$
R_{a b}+\frac{(d-1)}{l^{2}} g_{a b}=\partial_{a} \phi \partial_{b} \phi,
$$

where factors of $8 \pi G$ are suppressed. We assume the static spherically symmetric form

$$
d s^{2}=-g(r) h(r) d t^{2}+\frac{h(r)}{g(r)} d r^{2}+r^{2} d \Omega_{d-2}^{2},
$$

of the metric in $d$ spacetime dimensions, which give the coupled equations

$$
\begin{aligned}
r l^{2} g^{\prime}+(d-3)(g-h) l^{2}-(d-1) r^{2} h & =0, \\
(\ln h)^{\prime} & =\frac{r}{d-2}\left(\phi^{\prime}\right)^{2}, \\
\phi^{\prime} & =\frac{k}{g r^{d-2}},
\end{aligned}
$$

where the prime denotes the derivative with respect to $r$ and $k$ is an integration constant obtained by integrating the KleinGordon equation.

By a field redefinition $g(r):=\psi(r) / r^{d-3}$, the above set of equations give

$$
\psi^{2}\left[r \frac{\psi^{\prime \prime}}{\psi^{\prime}}-\frac{(d-1)(d-2) r^{2}+(d-3)(d-4) l^{2}}{(d-1) r^{2}+(d-3) l^{2}}\right]=\frac{k^{2}}{d-2} .
$$

This basic equation determines the spacetime metrics of interest. This equation is especially simple for $d=3$ and we deal with it separately, followed by the cases $d \geqslant 4$.

$d=3$. The differential equation (4) reduces to [12]

$$
g(r)^{2}\left[r \frac{g^{\prime \prime}(r)}{g(r)}-1\right]=k^{2} .
$$

The complete solution in this case can be expressed as $r$ $=r(g)$ with

$$
r=B l\left(g^{2}+A g / 2-k^{2} / 2\right)^{1 / 4}\left(\frac{g+A / 4+C / 4}{g+A / 4-C / 4}\right)^{A / 4 C},
$$

where $A$ and $B>0$ are integration constants, and $C$ $=\sqrt{8 k^{2}+A^{2}}$. Without loss of generality, we can set $B=1$. It is convenient to define the variables $x=g+A / 4, b=C / 4, a$ $=A / C$, in terms of which Eq. (6) becomes

$$
r=l(x-b)^{(1-a) / 4}(x+b)^{(1+a) / 4} .
$$

Using Eq. (3) gives

$$
\begin{aligned}
& h(x)=\frac{x^{2}-b^{2}}{x-a b}(x-b)^{-(1-a) / 2}(x+b)^{-(1+a) / 2}, \\
& \phi(x)=\frac{1}{2} \sqrt{\frac{1-a^{2}}{2}} \ln \left(\frac{x-b}{x+b}\right) .
\end{aligned}
$$

Thus the scalar field is real for $a^{2} \leqslant 1$. The metric may be written in the new radial coordinate $x$ as

$$
\begin{aligned}
d s^{2}= & -(x-b)^{(1+a) / 2}(x+b)^{(1-a) / 2} d t^{2}+\frac{l^{2}}{4\left(x^{2}-b^{2}\right)} d x^{2} \\
& +l^{2}(x-b)^{(1-a) / 2}(x+b)^{(1+a) / 2} d \Omega_{d-2}^{2} .
\end{aligned}
$$

The Ricci scalar of the metric is

$$
R=-2 \frac{3 x^{2}-4 b^{2}+a^{2} b^{2}}{l^{2}\left(x^{2}-b^{2}\right)},
$$

which shows that there are curvature singularities at $x=$ $\pm b$, corresponding to the origin $r=0$. Also, it confirms that the spacetime is AAdS, since $R(x \rightarrow \infty)=-6 / 1^{2}$. Since the solution contains no horizons for non-vanishing scalar field, the singularity at $r=0$ is naked.

There are two special cases of this metric which are familiar, both of which correspond to vanishing scalar field $k$ $=0$. The first is $a^{2}=1$ for which the metric reduces to

$$
d s^{2}=-\left(\frac{r^{2}}{l^{2}} \mp 2 b\right) d t^{2}+\frac{d r^{2}}{r^{2} / l^{2} \mp 2 b}+r^{2} d \theta^{2},
$$

where the $\mp$ signs correspond to $a= \pm 1$ respectively. Thus $a=1$ is the non-rotating BTZ black hole with mass $2 b$ $=C / 2$. The second is $b=0$ and $a$ arbitrary, which gives the zero mass BTZ black hole, rather than global $\mathrm{AdS}_{3}$ spacetime.

$d \geqslant 4$. For spacetime dimensions greater than three, Eq. (4) cannot be solved analytically. However it is possible to obtain an asymptotic expansion of the solution for large $r$. For $r \gg l$ (and fixed $l$ ), Eq. (4) can be approximated as

$$
\psi^{2}\left[\frac{\psi^{\prime \prime}}{\psi^{\prime}} r-(d-2)\right]=\frac{k^{2}}{d-2},
$$

which has the exact solution $\psi_{0}(r)$ given implicitly by

$$
\begin{aligned}
r= & B l^{2 /(d-1)}\left[\psi_{0}^{2}+\frac{A \psi_{0}}{d-1}-\frac{k^{2}}{(d-1)(d-2)}\right]^{1 / 2(d-1)} \\
& \times\left[\frac{\psi_{0}+A / 2(d-1)+C / 2(d-1)(d-2)}{\psi_{0}+A / 2(d-1)-C / 2(d-1)(d-2)}\right]^{(A / 2 C)[(d-2) /(d-1)]} .
\end{aligned}
$$

As before the metric for large $r$ may be written using the variables $x=\psi_{0}+A / 2(d-1), b=C / 2(d-1)(d-2)$, and $a$ $=(d-2) A / C$. For large $r, \psi_{0}(r) \sim r^{(d-1)}$.

The next term in the asymptotic expansion for large $r$ is obtained by writing 


$$
\psi(r)=\psi_{0}(r)\left(1+\alpha l^{2} / r^{2}\right),
$$

where $\alpha$ is a constant. For $d=5$ one finds $\alpha=1$ by substituting this expression into Eq. (4), a result which is useful for calculating conserved charges.

For AAdS geometries, the calculation of conserved charges is complicated by the occurrence of divergent expressions. These occur essentially because the metric diverges as $r^{2}$ for large $r$. There exist two quite distinct procedures for obtaining "regularized" finite expressions for asymptotic conserved charges. These are the so-called "conformal" [6] and "counter-term" [7] methods.

In the conformal method, a conformal transformation $g_{a b}=\Omega^{2} \hat{g}_{a b}$ is performed on the physical spacetime $\left(\hat{M}, \hat{g}_{a b}\right)$ under consideration, such that the asymptotic regions get mapped to a finite distance in a new manifold $\left(M, g_{a b}\right)$ [6]. A boundary is then added to this conformally transformed (and unphysical) manifold. This is especially useful for AAdS spacetimes, because many of the canonical metric components diverge asymptotically and limits such as $r \rightarrow \infty$ become rather tricky. On the other hand, the transformed manifold has a completely well-behaved structure.

The procedure basically involves showing that the electric parts of the Weyl tensor satisfy, as a consequence of Einstein's equations, a conservation law at null infinity, $\mathcal{I}$, in the conformally transformed spacetime. The end result is that the following equation holds on $\mathcal{I}$ :

$$
D^{p} E_{m p}=-8 \pi G \frac{(d-3)}{l} T_{a b} n^{a} h^{b}{ }_{m},
$$

where $D^{p}$ is the intrinsic covariant derivative on $\mathcal{I}$, compatible with the induced metric $h_{a b}:=g_{a b}-l^{2} n_{a} n_{b} \quad\left(n_{a}\right.$ $\left.:=\nabla_{a} \Omega\right), E_{a b}$ is the electric part of the Weyl tensor at $\mathcal{I}$ defined as $E_{a b}:=l^{2} \quad \Omega^{3-d} C_{a m b n} n^{m} n^{n}$ and $T_{a b}:=\Omega^{2-d} \hat{T}_{a b}$ on $\mathcal{I}$. The above conservation equations dictate the following form of conserved charge associated with the conformal Killing vector field $(\mathrm{KVF}) \xi$ :

$$
Q_{\xi}[C]:=-\frac{1}{8 \pi G} \frac{l}{d-3} \quad \oint_{C} E_{a b} \xi^{a} d S^{b}
$$

(An ordinary KVF on $\hat{M}$ becomes the conformal KVF on M.) In the presence of matter fields, this charge satisfies the covariant balance equation

$$
Q_{\xi}\left[C_{2}\right]-Q_{\xi}\left[C_{1}\right]=\int_{\Delta \mathcal{I}} T_{a b} \xi^{a} d S^{b}
$$

where $C_{1}$ and $C_{2}$ are two cross-sections on $\mathcal{I}$ bounding the region $\Delta \mathcal{I}$. Equations (15) and (16) are the fundamental relations which we will use to define conserved quantities. Thus apart from volume factors, the electric part of the Weyl tensor is the relevant quantity to be calculated. For our metrics the scalar field vanishes too quickly to be captured on the right hand side of Eq. (16).
TABLE I. Conserved quantities in the two approaches.

\begin{tabular}{lcc}
\hline \hline $\operatorname{Dim}$ & $Q_{1}$ & $Q_{2}$ \\
\hline 3 & - & $a b / 4 G$ \\
\hline 4 & $a b / G$ & $a b / G$ \\
\hline 5 & $9 \pi a b / 16 G$ & $9 \pi a b / 16 G+3 \pi l^{2} / 32 G$ \\
\hline \hline
\end{tabular}

The counter-term method proposes that the EinsteinHilbert action $S_{E H}$ should be supplemented with additional boundary terms dependent on the intrinsic metric $\gamma$. Since the variational principle is defined with fixed boundary metric, this does not change the equations of motion. The full action $S+S_{c t}$ is used to obtain an effective energy momentum tensor associated with the boundary,

$$
T_{a b}:=\frac{2}{\sqrt{-\gamma}} \frac{\delta\left(S_{E H}+S_{c t}\right)}{\delta \gamma^{a b}}
$$

The conserved charge associated with the symmetry generated by a vector field $\xi$ is then defined by

$$
Q_{\xi}:=\frac{1}{8 \pi G} \int_{\Sigma} d^{d-2} \sqrt{\sigma} T_{a b} u^{a} \xi^{b}
$$

where $\Sigma$ is a spatial slice of $\partial M, u^{a}$ is the timelike unit normal at $\Sigma$, and $\sigma_{a b}:=\gamma_{a b}+u_{a} u_{b}$.

For $d=3,4$ and 5, the following expression suffices to yield finite charges for commonly encountered AAdS spacetimes, including the scalar solutions:

$$
L_{c t}=-\frac{d-2}{l} \sqrt{-\gamma}-\frac{l \sqrt{-\gamma}}{2(d-3)} R(\gamma) .
$$

The resulting stress-energy tensor is

$$
\begin{aligned}
T_{a b}= & K_{a b}-\gamma_{a b} K-\frac{d-2}{l} \gamma_{a b} \\
& +\frac{l}{d-3}\left(R_{a b}(\gamma)-\frac{1}{2} \gamma_{a b} R(\gamma)\right) .
\end{aligned}
$$

The conserved charges for various dimensions are calculated using Eq. (15) in the conformal method $\left(Q_{1}\right)$, and Eq. (18) in the counterterm method $\left(Q_{2}\right)$. Restoring the $8 \pi G$ factors we obtain the expressions given in Table I.

Note that the conformal method does not apply for $d=3$, as the Weyl tensor is identically zero. The following comments discuss additional aspects of the solutions:

(i) Although we have not been able to find an exact solution of Eq. (4) above three dimensions for all $r$, there is strong evidence that the solutions in higher dimensions have curvature singularities at the origin. This may be seen analytically and numerically. Analytically, Eq. (4) can be integrated near $r=0$. For example, in $d=5$ this equation gives 


$$
\psi=\sqrt{\frac{k^{2}}{6}-\frac{r^{4}}{6 l^{4}}},
$$

from which the Ricci scalar is $R \sim r^{-6}$. Numerically, this equation can be integrated for all $r$ and $d$ (for example using MAPLE) [13]. For all initial conditions considered, $\phi^{\prime}$ shows a strong divergence at the origin, which is similar to the behavior seen analytically in three dimensions. Finally, an exact scalar field solution in five dimensions has been found recently by one of us [14]. Although this exact solution arises in different coordinates, it has the same symmetries as the class considered in this paper. This provides further evidence that the spherically symmetric scalar field-AdS spacetimes generically have naked singularties at the origin.

(ii) The two approaches for calculating conserved charges give positive energies for our scalar field solutions in spite of the fact that the solutions contain a naked singularity at the origin. This is unlike the negative mass Schwarzschild naked singularity, where the associated conserved charge is negative.

(iii) Except in $d=5$, the two approaches yield identical results. In $d=5$, the counterterm prescription predicts an additional Casimir energy, which is identical to that found in the context of AdS/CFT correspondence, and is independent of the scalar charge $k$. This is unlike AAdS spacetimes with rotation, where the vacuum energy depends on the rotation parameter [6]. Our results can be generalized to spacetime dimensions greater than five; the Casimir energy appears for all odd dimensional spacetimes.

(iv) In terms of the scalar field strength $k$, the conserved charges are proportional to

$$
a b=\frac{1}{4} \sqrt{\frac{C^{2}}{(d-2)^{2}}-4 k^{2} \frac{(d-1)}{(d-2)}}
$$

Note that the $k=0$ case is the AdS-Schwarzschild solution with mass $M=C /(d-2)$. Thus, surprisingly the presence of the scalar field effectively reduces the energy of the gravitational solution.

(v) There is an unexpected and interesting result in the "extremal" case

$$
\frac{C}{d-2}=2 k \sqrt{\frac{d-1}{d-2}} .
$$

The conserved charge vanishes in the conformal calculation for all dimensions and is therefore equal to the energy of global AdS in this method. The same holds for the counterterm method, except that the energies of the two solutions now equal the Casimir energy in five dimensions. This surprising degeneracy of energy associated with two distinct solutions on the gravity side raises an interesting question for the AdS/CFT conjecture: What effects on the CFT side distinguish global AdS from this extremal scalar field solution? It is possible that the answer lies in calculating other effects using the correspondence, such as n-point functions with these spacetimes as background (see below). (vi) The energy of Schwarzschild-AdS spacetime $M_{S A}$ (calculated using either method) can be matched by choosing $C$ and $k$ of the scalar solutions such that

$$
M_{S A}=\frac{1}{4} \sqrt{\frac{C^{2}}{(d-2)^{2}}-4 k^{2} \frac{(d-1)}{(d-2)}}
$$

Thus the energy degeneracy pointed out above, concerning global AdS, extends to the Schwarzschild-AdS metrics.

(vii) There exist certain intuitive criteria for the types of gravitational singularities that might be permitted in the context of the AdS/CFT conjecture [15]. The central singularity in the present solutions does not violate these. Thus it appears that an explanation of the degeneracy is not yet available.

(viii) Some evidence that the energy degeneracy discussed above may not be manifested in other calculations in the AdS/CFT context comes from consideration of the full action associated with our solutions. This computation can be done exactly in three dimensions. Surprisingly, the divergence at the origin does not make the action infinite. (Recall that the divergence for large $r$ is regulated by the subtraction procedure.) The key test is whether the scalar field parameter appears in the action. It does: $S=-\pi k$ (multiplied by a factor coming from the $t$ integration) for the "extremal" solution. (For comparison the action of global AdS is $-2 \pi$.) This situation is analagous to the result for Schwarzschild-AdS, where the action is a function of the black hole mass $M$. The natural interpretation of the latter $[16,3]$ is that it corresponds to a CFT at finite temperature. Since there is a scale in our solution, determined by the scalar field strength, the corresponding CFT must have conformal invariance broken. The exact nature of the breaking is apparently not due to temperature since temperature cannot be associated with naked singularities. Nevertheless, these considerations provide a clear distinction between global AdS and this class of scalar field spacetimes, regardless of the energy degeneracy.

In summary we have described solutions of general relativity with a cosmological constant coupled to a scalar field. In three spacetime dimensions, the solution is exact for all $r$, whereas for higher dimensions, the solution is an asymptotic one, for large $r$. The solutions have finite energy, although they do not possess an event horizon. The only nonzero charges are those associated with the timelike KVF. Furthermore, these charges contain information about the strength of the scalar field $k$. As we have discussed above, these results lead to interesting questions concerning the AdS/CFT conjecture. Among these are the issues of how a naked singularity on the gravity side translates to the field theory side, given that the associated energy is finite, and the meaning of the energy degeneracy of the extremal solution and global AdS. Finally, it would be interesting to see if the energy degeneracies we have found can lead to the possibility of phase transitions analagous to the Hawking-Page transition [17] between Schwarzschild-AdS black holes and global AdS.

This work was supported by the Natural Science and Engineering Research Council of Canada. 
[1] M.B. Green, J. Schwarz, and E. Witten, Superstring Theory (Cambridge University Press, Cambridge, England, 1988), Vols. I and II; J. Polchinski, String Theory (Cambridge University Press, Cambridge, England, 1998), Vols. I and II.

[2] M. Choptuik, Phys. Rev. Lett. 70, 9 (1993); for a review see C. Gundlach, Living Rev. Relativ. 2, 4 (1999).

[3] J.M. Maldacena, Adv. Theor. Math. Phys. 2, 231 (1998); for a review, see O. Aharony, S.S. Gubser, J. Maldacena, H. Ooguri, and Y. Oz, Phys. Rep. 323, 183 (2000).

[4] See for example D.Z. Freedman, S.D. Mathur, A. Matusis, and L. Rastelli, Nucl. Phys. B546, 96 (1999); W. Mueck and K.S. Viswanathan, Phys. Rev. D 58, 041901 (1998); S. Das and A. Dasgupta, J. High Energy Phys. 10, 025 (1999); G. Horowitz and V. Hubeny, ibid. 10, 027 (2000).

[5] A. Peet and J. Polchinski, Phys. Rev. D 59, 065011 (1999).

[6] A. Ashtekar and A. Magnon, Class. Quantum Grav. 1, L39 (1984); A. Ashtekar and S. Das, ibid. 17, L17 (2000); S. Das and R.B. Mann, J. High Energy Phys. 08, 033 (2000).

[7] M. Henningson and K. Skenderis, J. High Energy Phys. 07, 023 (1998); V. Balasubramanian and P. Kraus, Commun. Math. Phys. 208, 413 (1999); R.B. Mann, Phys. Rev. D 60, 104047 (1999); R. Emparan, C.V. Johnson, and R.C. Myers, ibid. 60, 104001 (1999); P. Kraus, F. Larsen and R. Siebelink, Nucl. Phys. B563, 259 (1999); A.M. Awad and C.V. Johnson, Phys. Rev. D 61, 084025 (2000).

[8] G. Horowitz and R. Myers, Gen. Relativ. Gravit. 27, 915 (1995).
[9] F. Pretorius and M.W. Choptuik, Phys. Rev. D 62, 124012 (2000).

[10] V. Husain and M. Olivier, Class. Quantum Grav. 18, L1 (2001).

[11] G. Clement and A. Fabbri, "Analytical treatment of critical collapse in 2+1 dimensional AdS spacetime," gr-qc/0101073.

[12] We learned that a similar equation is considered in L.M. Burko, Phys. Rev. D 62, 127503 (2000); the 3-dimensional solution is derived in G. Clement and A. Fabbri, Class. Quantum Grav. 17, 2537 (2000); see also S. Nojiri and S.D. Odintsov, Phys. Rev. D 61, 044014 (2000), and references therein for solutions in dilatonic gravity.

[13] Equation (4) may be integrated, for various dimensions and boundary conditions, using the following MAPLE code: with(plots): $\mathrm{k}:=2: \quad \mathrm{l}:=1: \quad \mathrm{d}:=5: \quad \mathrm{de}:=\{\mathrm{p}(\mathrm{r}) \hat{\wedge} 2 *(\mathrm{r} * \operatorname{diff}(\mathrm{p}(\mathrm{r}), \mathrm{r}, \mathrm{r})$ $/ \operatorname{diff}(\mathrm{p}(\mathrm{r}), \mathrm{r})-((\mathrm{d}-1) *(\mathrm{~d}-2) * \hat{\mathrm{r}} 2+(\mathrm{d}-3) *(\mathrm{~d}-4) * 1 \hat{2}) /((\mathrm{d}-1) * \hat{\mathrm{r}} 2$ $+(\mathrm{d}-3) * l \hat{2}))-\mathrm{k} 2 / 3\}$ : init $:=\{\mathrm{p}(10)=1, \mathrm{D}(\mathrm{p})(10)=1\}: \mathrm{psi}$ $:=$ dsolve(de union init, $\mathrm{p}(\mathrm{r})$, type $=$ numeric) : odeplot $(\mathrm{psi},[\mathrm{r}, \mathrm{k} /$ $(\mathrm{p}(\mathrm{r}) * \mathrm{r})], 0.1 . .10$, numpoints $=50)$.

[14] V. Husain, "Dualities and Wilson loops," Plenary Lecture in Proceedings of the 9th Canadian Conference on General Relativity and Relativistic Astrophysics; http:// www.math.ualberta.ca/ ccgrra

[15] S. Gubser, "Curvature singularities; the good, the bad and the naked," hep-th/0002160.

[16] E. Witten, Adv. Theor. Math. Phys. 2, 505 (1998).

[17] S.W. Hawking and D. Page, Commun. Math. Phys. 87, 577 (1983). 